\title{
Sacubitril/valsartan (LCZ696) reduces myocardial injury following myocardial infarction by inhibiting NLRP3-induced pyroptosis via the TAK1/JNK signaling pathway
}

\author{
JIANFEN SHEN ${ }^{1}$, ZHONGBAO FAN $^{2}$, GUANG SUN $^{3}$ and GUOXIAN QI ${ }^{3}$ \\ ${ }^{1}$ Department of Cardiology, The First Affiliated Hospital of China Medical University, Shenyang, \\ Liaoning 110001; ${ }^{2}$ Department of Hepatobiliary Surgery, People's Hospital of China Medical University, \\ Liaoning Provincial People's Hospital, Shenyang, Liaoning 110016; ${ }^{3}$ Department of Geriatric Cardiology, \\ The First Affiliated Hospital of China Medical University, Shenyang, Liaoning 110001, P.R. China
}

Received August 30, 2020; Accepted March 1, 2021

DOI: $10.3892 / \mathrm{mmr} .2021 .12315$

\begin{abstract}
The present study aimed to investigate the protective effects of sacubitril/valsartan (LCZ696) on ventricular remodeling in myocardial infarction (MI) and the effects of the inflammasome-mediated inflammatory response. First, a rat model was established. Animals were then treated with LCZ696 so that the histopathological changes associated with ventricular remodeling could be investigated. The serum levels of the inflammatory factors IL-18 and IL-1 $\beta$ were also determined by ELISA. Immunofluorescence was used to investigate the ratio of pyroptosis following MI modelling. Western blotting and reverse transcription-quantitative PCR were used to detect the relative expression levels of proteins and mRNAs in the transforming growth factor $\beta$-activated kinase-1 (TAK1)/JNK pathway and those associated with the NLR pyrin family domain containing 3 (NLRP3) inflammasome, respectively. The present study also investigated the regulatory mechanisms and associations between the TAK1 and JNK pathways, NOD-, leucine-rich repeat- and the NLRP3 inflammasome, in H9C2 cells and myocardial cells from the rat model of MI. LCZ696 improved MI-induced myocardial fibrosis, rescued myocardial injury and suppressed the release of inflammatory factors. With regards to myocardial cell damage, pyroptosis in cardiomyocytes was observed. The in vitro experiments demonstrated that the overexpression of TAK1 promoted lysis of the N-terminal of GSDMD, thereby activating the NLRP3 inflammasome and promoting the conversion of pro-IL-1 $\beta$ and pro-IL-18 into mature IL-1 $\beta$ and IL-18, respectively. In contrast, the silencing of TAK1
\end{abstract}

Correspondence to: Dr Guoxian Qi, Department of Geriatric Cardiology, The First Affiliated Hospital of China Medical University, 155 Nanjing North Street, Heping, Shenyang, Liaoning 110001, P.R. China

E-mail: qigx@cmulh.com

Key words: LCZ696, myocardial infarction, NLRP3 inflammasome, TAK1/JNK signaling pathway inhibited the expression levels of the NLRP3 inflammasome. In summary, LCZ696 reduced the expression levels of the NLRP3 inflammasome, suppressed inflammatory responses, improved the ventricular remodeling and exhibited protective effects in the MI heart by inhibiting the TAK1/JNK signaling pathway.

\section{Introduction}

Myocardial infarction (MI) is a common disease of the cardiovascular system (1). Apoptosis is a distinctive mode of programmed cell death (2). There is a causal connection between cardiac myocyte apoptosis and MI (1). MI can cause a progressive decline in cardiac function that can develop into heart failure (3). Pyroptosis is an important form of programmed cell death and is characterized by cellular swelling, membrane disruption and the release of pro-inflammatory cytoplasmic contents (4). The induction of cysteine aspartic proteolytic enzyme (caspase) is known to induce pyroptosis and inflammatory responses (4). Previous studies have focused on the process of pyroptosis in a range of different heart diseases $(5,6)$. However, the investigation of pyroptosis in MI remains to be elucidated.

Ventricular remodeling is an important pathological process underlying MI. The severity of ventricular remodeling is known to determine the prognosis of patients with MI (7). The death of myocardial cells is an important factor in the process of ventricular remodeling and represents the primary cause of cardiac dysfunction (8). The progress and development of ventricular modelling may lead to mortality in patients with MI (7). Since myocardial cells mainly die during the early stages of ventricular remodeling (9), preventing the death of myocardial cells during the early stages of ventricular remodeling following MI is a key target if MI-induced ventricular remodeling is to improve.

TGF- $\beta$ serves an important role in the process of ventricular remodeling and can target myocardial cells by activating TGF- $\beta$-activated kinase 1 (TAK1) and its downstream factor, JNK (10-12). TAK1 is involved in the differentiation of cardiomyocytes and the growth and development of the heart; 
consequently, TAK1 is important in cardiac disease (13). The study demonstrates that TAK1 regulates cell viability and inflammatory responses by activating its downstream effectors: NF- $\kappa \mathrm{B}$ and MAPK (14). Okada et al (15) firstly proposed that the TAK1/JNK pathway acts as an essential regulator for the activation of the NLRP3 inflammasome.

It has been confirmed that expression levels of NLRP3 are increased in the early stages of ventricular remodeling following MI and that the combination of NLRP3 with apoptosis-associated speck-like protein containing a caspase-recruitment domain (ASC) can recruit and activate caspase-1 thus promoting the maturation and release of IL-1 $\beta$. IL-1 $\beta$, an important inflammatory molecule, recruits nearby inflammation-related cells following MI, thus amplifying the inflammatory response and participating in post-MI ventricular remodeling, eventually leading to the death of myocardial cells (16-19). Another study demonstrated that the specific inhibition of IL- $1 \beta$ can significantly improve the contractile function of a mouse model of MI mice and can also reduce enlargement of the heart; these data suggested that early ventricular remodeling might be related to cellular pyroptosis (20).

Pyroptosis is an inflammasome-activated process. Caspase-1-mediated pyroptosis refers to a process that takes place in inflammasomes but is activated by caspase-1 (21). The specific blocking of the NLRP3 inflammasome with ASC markedly reduces cardiomyocyte death and improves cardiac function, thus indicating that NLRP3 inflammasomes are involved in the early stages of ventricular remodeling following MI (22-24).

Sacubitril/valsartan (LCZ696) is a new form of neuroendocrine inhibitor that can selectively and efficiently inhibit the angiotensin II (Ang II) receptor and enkephalinase (25). Ang II is not only a strong vasoconstriction and growth-promoting peptide; research has shown that this peptide is closely associated with cardiac remodeling and can promote hypertrophy and fibrosis in cardiomyocytes (26-28). In a previous study, Kompa et al (29) established a rat model of MI and treated these animals with LCZ696 and perindopril. The study demonstrated that the left ventricular ejection fraction and shortened fraction of rats under LCZ696 treatment are enhanced compared with those rats treated with perindopril; furthermore, LCZ696 exhibits a more powerful effect in terms of myocardial protection (29). Perindopril, an ACE inhibitor and amlodipine, a dihydropyridine calcium channel blocker, is known to be able to treat cardiovascular events $(30,31)$. In a previous clinical trial, LCZ696 significantly reduced the risk of cardiovascular death and hospitalization of patients experiencing heart failure when compared with enalapril; these effects were due to the inhibition of TGF- $\beta$ and the consequential reduction in ejection fraction and improvement in cardiac function. Thus, LCZ696 represents a new alternative treatment option for chronic heart failure compared with the traditional treatment of renin-angiotensin-aldosterone system (RAAS) blockade (32).

The present study established a rat model of MI and the animals were treated with LCZ696 to explore its protective effects on the heart and the inflammasome-mediated inflammatory response. The present study also aimed to investigate the regulatory mechanisms associated with LCZ696 that link the TAK1/JNK pathway and NLRP3 inflammasomes.

\section{Materials and methods}

Experimental animals. A total of 72 male 3-month-old SPF-grade Sprague-Dawley (SD) rats, weighing 260-300 g, were purchased from VitalRiver Experimental Animal Techniques [Production License: SCXK (Beijing) 20190001]. All animal were kept in a barrier room with free access to food and water under 12-h light/dark cycle and 40-70\% humidity at $20-25^{\circ} \mathrm{C}$ at Department of Laboratory Animal Science of China Medical University (User License: SYXK (Liao) 2013001). Rats were randomly divided into the following groups: Sham operation group (Sham group, $n=24)$, myocardial infarction model group (MI group, n=24) and a MI + LCZ696 treatment group (LCZ696 group, $n=24$ ). The experiments were approved by the Laboratory Animal Welfare and Ethics Committee of China Medical University (approval. number IACUC NO. 2019109).

Establishment of a rat model of myocardial infraction. Rats were anaesthetized with intraperitoneal injection of $1 \%$ pentobarbital sodium $(50 \mathrm{mg} / \mathrm{kg})$. Electrocardiogram parameters were recorded using BL-420 biological and functional experimental system (TECHMAN). Respiratory support was provided by a small animal ventilator. Next, the skin of each rat was cut in a longitudinal manner from the left margin of the 3rd to 4th intercostal space to expose the heart. The left anterior descending coronary artery was then ligated $\sim 3-4 \mathrm{~mm}$ below the intersection of the left atrial appendage root and the pulmonary artery conical clip. When ligated in this manner, the myocardial tissue began to turn white and the ST segment of the electrocardiogram demonstrated different levels of elevation. Collectively, these indicators demonstrated that the rat model had been successfully established. Rats in the Sham group were treated by thoracotomy only while rats in the LCZ696 group were administered with $68 \mathrm{mg} / \mathrm{kg}$ LCZ696 (SML1380; Sigma-Aldrich; Merck KGaA) by oral gavage daily for a total period of 7 days after the rat model of MI had been established (33).

Examining left ventricular function. A total of eight rats from each group underwent cardiac ultrasonic examination (EPIQ7C; Philips Medical Systems B.V.) to analyze left ventricular (LV) function at each detection time point $(1,3$ and 7 days). Rats were first anaesthetized with $1 \%$ sodium pentobarbital $(50 \mathrm{mg} / \mathrm{kg})$; the fur on the left side of the chest was then removed using hair removal cream. M-mode ultrasound was then used to detect the LV ejection fraction (LVEF), LV fractional shortening (LVFS), LV internal diameter at systole (LVIDs) and LV diastolic diameter (LVIDd).

Sample collection. At each detection time point (1, 3 and 7 days), eight rats from each experimental group were randomly selected for blood and tissue sample collection. First, rats were placed on an operating table for anesthetized with $1 \%$ sodium pentobarbital $(50 \mathrm{mg} / \mathrm{kg})$. The abdominal cavity was then opened and the abdominal aorta was cut to acquire $2 \mathrm{ml}$ samples of blood. Blood samples were stored in $-80^{\circ} \mathrm{C}$ freezer for subsequent ELISA. Following euthanasia through overdose of $1 \%$ sodium pentobarbital $(200 \mathrm{mg} / \mathrm{kg})$, the chest cavity was opened to collect samples of heart tissue. Half of 
each heart tissue sample was immersed in $10 \%$ formalin and fixed for $24 \mathrm{~h}$ at room temperature for subsequent staining to investigate pathological changes. The other half of each heart tissue sample was stored in a $-80^{\circ} \mathrm{C}$ freezer to await protein detection.

Determining the size of the infarction. 2,3,5-Triphenyltetrazolium chloride (TTC) staining was used to determine the size of the myocardial infarct. Thin sections of heart tissue were prepared from each sample collected on the day 1, 3 and 7. Briefly, the rat heart was frozen quickly in liquid nitrogen and cut into $2 \mathrm{~mm}$ sections. The sections were incubated with $2 \%$ TTC staining solution at $37^{\circ} \mathrm{C}$ for $30 \mathrm{~min}$. Post-staining, the tissue slices were fixed overnight in $10 \%$ formalin solution at $4^{\circ} \mathrm{C}$. Representative images were then captured from each tissue section using a digital camera. The area of infarction within the sections of heart tissue (which remained unstained following incubation with TTC staining solution) was then determined by Sigma Scan Pro 5.0 software (Systat Software, Inc.).

Hematoxylin and eosin $(H \& E)$ staining. Post-perfused heart tissues were collected from sacrificed rats on days 1, 3 and 7 and fixed in neutral formalin; these tissues were then dehydrated with ethanol using a gradient series of concentrations $(75,80,95$ and $100 \%)$. The tissues were treated with xylene to clear them, embedded in paraffin and then cut into $4 \mu \mathrm{m}$ sections. For H\&E staining, sections were deparaffinized, stained first with hematoxylin and then stained with eosin at room temperature. The experiment procedure was according to the manufacturer's instructions (G1120,Solaibio,China) Histopathological changes in the myocardium were then evaluated by light microscopy.

\section{Temperature and duration of staining}

Masson's staining. A Masson's trichrome staining kit (Solarbio, G1345) was used according to the manufacturer's instructions at room temperature. Deparaffinized myocardium sections were stained in hematoxylin for $3 \mathrm{~min}$, differentiated in picric acid ethanol for $10 \mathrm{sec}$ and were then rinsed with distilled water. Sections were then stained blue by applying Masson's blueing solution for $3 \mathrm{~min}$, followed by $60 \mathrm{sec}$ rinse in distilled water. The sections were then stained in ponceau magenta solution for $5 \mathrm{~min}$ and rinsed with $2 \%$ glacial acetic acid aqueous solution. Following this, sections were washed with phosphomolybdic acid solution for $3 \mathrm{~min}$, stained in aniline blue for $3 \mathrm{~min}$ and differentiated in $1 \%$ glacial acetic acid. Finally, sections were dehydrated three times with pure ethanol ( $5 \mathrm{~min} /$ incubation), cleared with xylene for $5 \mathrm{~min}$ and then mounted in neutral resin. Slides were then evaluated by light microscopy. Myocardial collagen volume fraction (CVF) was analyzed by Image-Pro Plus 6.0 (Media Cybernetics, Inc.). CVF was calculated using the formula shown in Equation (1). Equation (1):

\section{$\mathrm{CVF}=($ Area of myocardial collagen/Total area $) \times 100 \%$}

ELISA. The serum levels of IL-18 (cat. no. SEA064Ra; Wuhan USCN Business Co., Ltd.) and IL-1 $\beta$ (cat. no. SEA563Ra; Wuhan USCN Business Co., Ltd.) were detected on days 1,
3 and 7 by ELISA in accordance with the manufacturer's guidelines. Blood samples were collected from the abdominal aorta of euthanized rats. In brief, $100 \mu \mathrm{l}$ of standard solutions and diluted samples were loaded into each well of the ELISA plates and incubated at $37^{\circ} \mathrm{C}$ for $1 \mathrm{~h}$. Detection reagent $\mathrm{A}$ and $\mathrm{B}$ were added and then the plates rinsed with the wash buffer provided in the ELISA kits. After thorough rinsing, $90 \mu \mathrm{l}$ of TMB substrate was added into each well and incubated at $37^{\circ} \mathrm{C}$ for $20 \mathrm{~min}$ in the dark. Stop solution was then applied to the plates and the optical density of each well was detected on a microplate reader at $450 \mathrm{~nm}$ within $10 \mathrm{~min}$ of adding the stop solution. The concentration of IL-18 and IL-1 $\beta$ in mice sera were calculated by a formula obtained from the standard curve.

Immunofluorescence. Heart tissue sections were prepared from days 1, 3 and 7 and then deparaffinized, rehydrated, incubated in $3 \% \mathrm{H}_{2} \mathrm{O}_{2}$ solution and then rinsed with PBS buffer. Sodium citrate solution $(0.1 \mathrm{M})$ was applied to the sections for antigen retrieval and then the sections blocked with goat serum at $37^{\circ} \mathrm{C}$ for $30 \mathrm{~min}$. Following incubation, the residual goat serum was decanted and then the sections incubated overnight at $4^{\circ} \mathrm{C}$ with a far-red dye labelled reactive oxygen species (ROS) probe (cat. no. C10422; Invitrogen; Thermo Fisher Scientific, Inc.) and primary antibodies against TAK1 (1:1,000; cat. no. PA5-20083; Invitrogen; Thermo Fisher Scientific, Inc.), phosphorylated (p)-JNK (1:1,000; cat. no. MA5-15228; Invitrogen; Thermo Fisher Scientific, Inc.), NLRP3 (1:1,000; cat. no. PA5-79740; Invitrogen; Thermo Fisher Scientific, Inc.) and gasdermin D (1:500; GSDMD; cat. no. NBP2-80427; Novus Biologicals, LLC). The sections were then rinsed with PBS buffer, incubated with fluorescence-labelled secondary antibody at $37^{\circ} \mathrm{C}$ for $30 \mathrm{~min}$ and rinsed thoroughly with PBS buffer. Cell nuclei were then incubated with DAPI (cat. no. D3571; Invitrogen; Thermo Fisher Scientific, Inc.) at room temperature for $10 \mathrm{~min}$. Finally, the sections were rinsed in PBS and sealed with anti-fade working solution. Expression levels were then evaluated with a fluorescent microscope and quantified with Image J 1.52q software (National Institutes of Health).

Western blotting. Frozen myocardial tissues were removed from the $-80^{\circ} \mathrm{C}$ freezer, thawed and crushed. After that, $1 \mathrm{ml}$ of RIPA lysis buffer (cat. no. 89901; Thermo Scientific, Inc.) containing $1 \%$ PMSF was added and total protein extracted by homogenizing and centrifuging the tissue for $15 \mathrm{~min}$ at $15,000 \mathrm{x} \mathrm{g}$ and $4^{\circ} \mathrm{C}$. The concentration of total protein extracts in the supernatants was then determined with a BCA assay kit (cat. no. 23227; Thermo Scientific, Inc.). Protein samples $(30 \mathrm{mg})$ were then separated by $10 \%$ SDS-PAGE and transferred on to $0.45 \mu \mathrm{m}$ PVDF membranes. Membranes were then blocked for $2 \mathrm{~h}$ with $5 \%$ skimmed milk at room temperature. Next, membranes were incubated overnight at $4^{\circ} \mathrm{C}$ with primary antibodies against TAK $1(1: 1,000$; cat.no. 4505; Cell Signaling Technology, Inc.), p-JNK (1:1,000; cat. no. 9255; Cell Signaling Technology, Inc.), JNK (1:1,000; cat. no. 9252; Cell Signaling Technology, Inc.), NLRP3 (1:1,000; cat. no. ab263899; Abcam), pro-caspase1 (1:1,000; cat. no. ab179515; Abcam), GSDMD (1:500; cat no. 93709; Cell Signaling Technology, Inc.), N-terminal cleavage product (GSDMD-NT) (1:1,000; cat. no. 93709; Cell Signaling 
Technology, Inc.), pro-IL-1 $\beta$ (1:1,000; cat. no. ab205924; Abcam) and pro-IL-18 (1:1,000; cat. no. ab191860; Abcam); GAPDH (1:2,000, cat. no. ab181602; Abcam) was used as an internal loading control. Residual primary antibodies were removed by washing the membranes three times in TBS-Tween $20(0.1 \%)$ on a shaking incubator. HRP-conjugated goat anti-rabbit secondary antibody (cat. no. 7074; Cell Signaling Technology, Inc.) was applied to the membranes and incubated for $1 \mathrm{~h}$ at room temperature. The membranes were then washed three times in TBS-Tween 20 and imaged using an imaging system and ECL chemiluminescent substrate (cat. no. 34580; Thermo Scientific, Inc.). Finally, grey values were analyzed using Image J $1.52 \mathrm{q}$ software (National Institutes of Health).

Reverse transcription-quantitative $(R T-q) P C R$. The experiments of RNA extraction, cDNA synthesis and qPCR were performed according to the manufacturer's protocols. Myocardial tissues were recovered from $-80^{\circ} \mathrm{C}$ and mixed with $1 \mathrm{ml}$ of TRIzol ${ }^{\circledR}$ (cat. no. 15596026; Thermo Fisher Scientific, Inc.). Samples were then thoroughly homogenized at low temperature to extract total RNA. cDNA was synthesized by reverse transcription and RT-qPCR performed using primers designed against TAKI and JNK; GAPDH was used as an internal control (Table I). RT-qPCR was carried out with a SYBR Primer-Script RT-PCR kit (Takara Biotechnology Co., Ltd.) using the following cycle parameters (for 40 cycles): pre-denaturation $\left(95^{\circ} \mathrm{C}, 30 \mathrm{sec}\right.$ ), annealing $\left(95^{\circ} \mathrm{C}, 5 \mathrm{sec}\right)$, extension $\left(60^{\circ} \mathrm{C}, 20 \mathrm{sec}\right)$. The relative expression levels of each gene were then determined using the $2^{-\Delta \Delta c t}$ method (34). All RT-qPCR experiments were repeated at least three times.

Cell culture. $\mathrm{H} 9 \mathrm{C} 2$ cells were cultured in a hypoxic culture chamber $\left(37^{\circ} \mathrm{C}, 2.5 \% \mathrm{O}_{2}, 5 \% \mathrm{CO}_{2}, 92.5 \% \mathrm{~N}_{2}\right)$ to establish a cellular model of MI (35). Cells in the control group were cultured in a normal culture chamber $\left(37^{\circ} \mathrm{C}, 5 \% \mathrm{CO}_{2}\right)$. Cells were cultured with DMEM medium (cat. no. 12491015; Gibco; Thermo Fisher Scientific, Inc.) without serum. TAK1 overexpression plasmids were obtained from Mingshanshang Medical Biotechnology Co., Ltd. and validated by PCR, enzymic digestion and DNA sequencing. Next, $500 \mathrm{ng}$ of DNA was transfected into cells using Lipofectamine ${ }^{\circledR} 3000$ (cat. no. L3000001; Invitrogen; Thermo Fisher Scientific, Inc.) and/or $20 \mu \mathrm{m}$ of LCZ696 was added to the cells transfected with the TAK1 overexpression plasmid, in appropriate with the following experimental groupings: Control group (negative control without plasmid transfection), hypoxia MI model group, vector + LCZ696 + hypoxia MI group and a TAK1 overexpression group + LCZ696 + hypoxia MI group. Experiments were replicated four times.

Statistical analysis. Data were represented as mean \pm standard deviation and analyzed using SPSS 21.0 (IBM Corp.). Multiple groups were compared by one-way analysis of variance. Tukey's post hoc was used to compare between groups at different time points (days 1, 3 and 7). The non-parametric Wilcoxon signed ranks test was used for multiple comparisons with adjusted P-values. $\mathrm{P}<0.05$ was considered to indicate a statistically significant difference.
Table I. Primers used for reverse transcription-quantitative PCR.

\begin{tabular}{ll}
\hline Gene & \multicolumn{1}{c}{ Primer } \\
\hline NLRP3 & F: TGAAGAGTGTGATCTGCGGAA \\
& R: GAAAGTCATGTGGCTGAAGCT \\
GSDMD & F: TGAATGTGTACTCGCTGAGTGT \\
& R: CAGCTGCTGCAGGACTTTGTG \\
Caspase-1 & F: CAGAGCTGTGCAGATGAGT \\
& R: CTGCAGCCACTGGTTCTGT \\
IL-1 $\beta$ & F: AGGAGAGACAAGCAACGACA \\
& R: CTTTTCCATCTTCTTCTTTGGGT \\
IL-18 & F: GCCATGTCAGAAGACTCTTGCGTC \\
& R: GTACAGTGAAGTCGGCCAAAGTTGTC \\
TAK1 & F: AGTAGGCCACATTACACTGCT \\
& R: GACCCACACCTCACAAATTGA \\
JNK & F: TCATGTGGTCAAGACGAGAT \\
& R: GGAGGAAGGAAGGCATGA \\
GAPDH & F: GCACACAGTACATCCGTCA \\
& R: TTCTCCGAACGTGTCACGT
\end{tabular}

NLRP3, NLR pyrin family domain containing 3; GSDMD, gasdermin D; F, forward; R, reverse.

\section{Results}

LCZ696 improves MI-induced myocardial damage and suppresses inflammatory responses. Cardiac ultrasound of LV function demonstrated that MI could lead to a thicker diameter of LV in both diastole and systole, thus resulting in a fraction reduction and a poorer ejection fraction (Fig. 1A); this effect was time-dependent. The size of the infarction was confirmed by TTC staining; the area of infarction increased significantly following MI (Fig. 1B). Inflammatory cell infiltration was observed in myocardial tissues following the occurrence of MI and cell degeneration was detected on day 3 following MI; these indicators had increased significantly by day 7 (Fig. 1C). There was no obvious interstitial fibrosis on days 1 and 3 following MI. However, obvious interstitial fibrosis was observed in the MI area and the area surrounding the MI area, on day 7 following MI (Fig. 1D). After seven days of LCZ696 treatment, a neat arrangement of myocardial cells was observed. The data demonstrated that LCZ696 reduced the level of interstitial fibrosis, leading to a significant reduction in CVF when compared to that in rats in the MI group on day 7 (Fig. 1E). It was found that inflammatory responses began on day 1 after MI; this involved a significant increase in the levels of IL-1 $\beta$ and IL-18. However, LCZ696 effectively reduced the relative levels of these inflammatory factors in the serum (Fig. 1F), suggesting that LCZ696 can improve the myocardial damage caused by MI and inhibit inflammation.

LCZ696 inhibits the expression of the NLRP3 inflammasome following MI. To investigate the inhibition of LCZ696 on NLRP3 inflammasome, it was found that the expression 

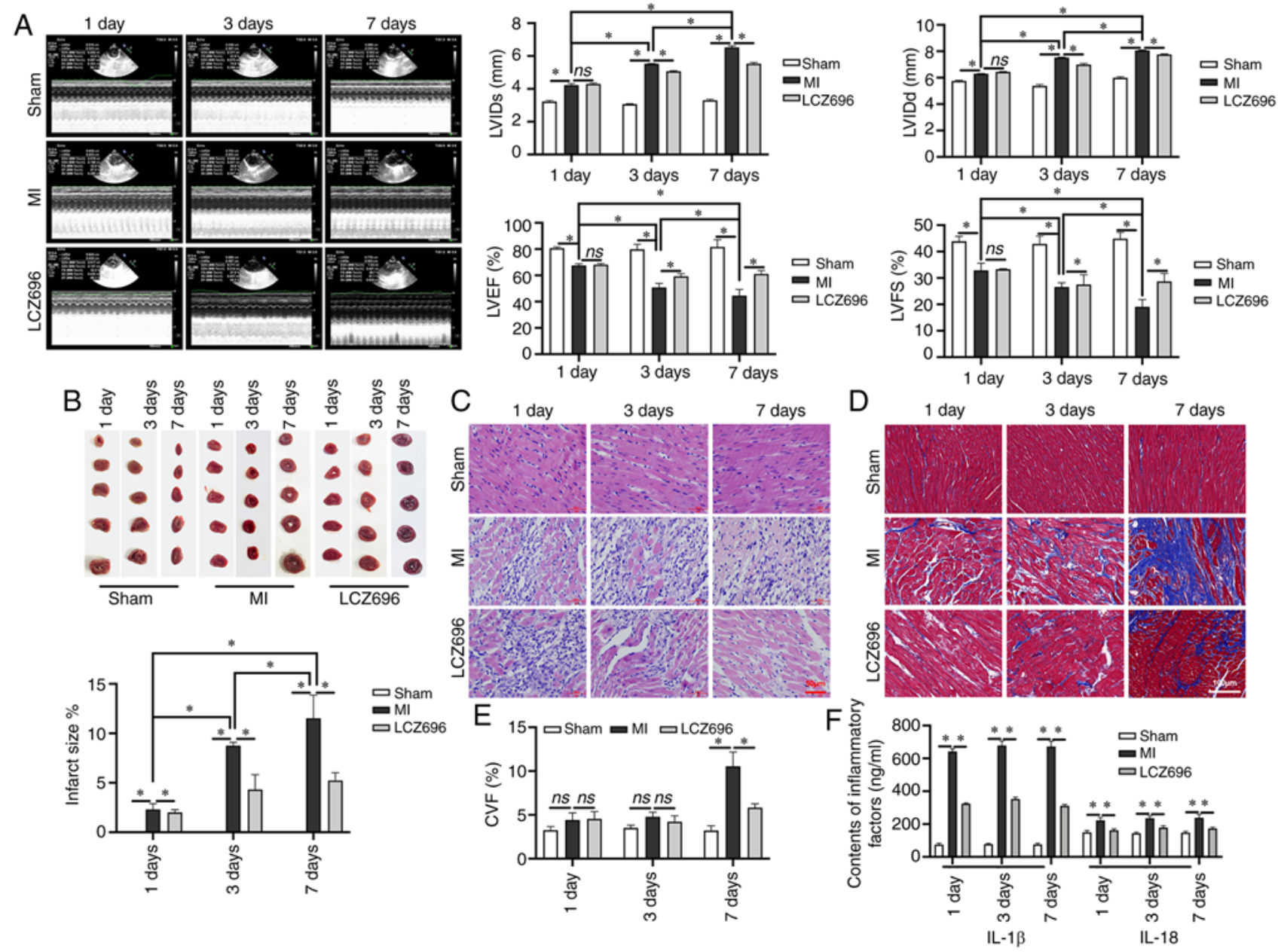

Figure 1. LCZ696 rescues MI-induced histopathological changes in myocardial cells and improves the inflammatory response in rats. (A) M-mode ultrasonic examination and LV function analysis ( $\mathrm{n}=24)$. (B) Infarct size, as detected by TTC staining. (C) H\&E staining of myocardial tissues (scale bar $=50 \mu \mathrm{m}$ ). (D) Interstitial fibrosis by Masson's staining (scale bar=100 $\mu \mathrm{m}$ ). (E) CVF (\%) as determined by Masson's staining. (F) Levels of IL-1 $\beta$ and IL-18 in the serum of experimental rats, as detected by ELISA. Data are given as mean \pm standard deviation, ${ }^{*} \mathrm{P}<0.05, n s ; \mathrm{P}>0.05$. LVIDs: left ventricular systolic internal dimension. LCZ696, sacubitril/valsartan; MI, myocardial infarction; TTC, triphenyltetrazolium chloride; H\&E, hematoxylin and eosin; CVF, collagen volume fraction; LVEF, left ventricular ejection fraction; LVFS, left ventricular fractional shortening, as determined by the following formula: (LVIDd-LVIDs)/LVIDd $\mathrm{x} 100 \%$; CVF was calculated as follows: $\mathrm{CVF}=$ collagen area/view area $\mathrm{x} 100 \%$.

levels of the NLRP3 inflammasome increased steadily from day 1 following MI (Fig. 2A and B). A significant accumulation of ROS following MI was also detected and the levels of ROS remained high until day 7 following MI (Fig. 2C). When NLRP3 was activated, the expression levels of pro-caspase-1 were upregulated and the expression levels of caspase-1 p10, pro-IL-1 $\beta$ and pro-IL-18, were also upregulated (Fig. 2D and E); these proteins were subsequently transformed into biologically activate IL-1 $\beta$ and IL-18 (Fig. 2F). LCZ696 was able to inhibit the accumulation of ROS and downregulate the expression levels of NLRP3. LCZ696 was also able to downregulate the expression levels of pro-caspase-1, pro-IL-1 $\beta$ and pro-IL-18 and suppress the release of IL-1 $\beta$ and IL-18.

MI mediates the extent of pyroptosis. In view of the clear association between NLRP3 inflammasome activation and pyroptosis, whether pyroptosis occurred after the establishment of MI model was next investigated. Pyroptosis is known as GSDMD-dependent programmed cell death; thus, GSDMD is a crucial executor of pyroptosis and is cleaved by activated inflammatory caspases- 1 into gasdermin
N-terminal (GSDMD-NT) and gasdermin C-terminal $(36,37)$. GSDMD-NT assembles membrane pores to promote cytolysis, thus leading to programmed necrosis/cell death, which is a key step in pyroptosis $(36,37)$. The present study found that the expression and lysis of GSDMD increased significantly from day 1 of MI and that LCZ696 could reduce these effects (Fig. 3A and B). These results demonstrated that pyroptosis might serve an important role in the progression of myocardial injury following MI.

LCZ696 downregulates the expression of the TAK1/JNK pathway-related proteins following MI. TAK1 is known to participate in the regulation of cell pyroptosis $(38,39)$ and may be activated by various intracellular and extracellular stimuli in order to regulate cell viability and inflammation. The present study investigated changes in the expression levels of key proteins in the TAK1/JNK pathway following MI and found that the protein and mRNA levels of TAK1, and the phosphorylation of JNK, were upregulated from day 1 following MI (Fig. 4A and B). It was also found that LCZ696 inhibited the expression of key proteins in the TAK1/JNK 


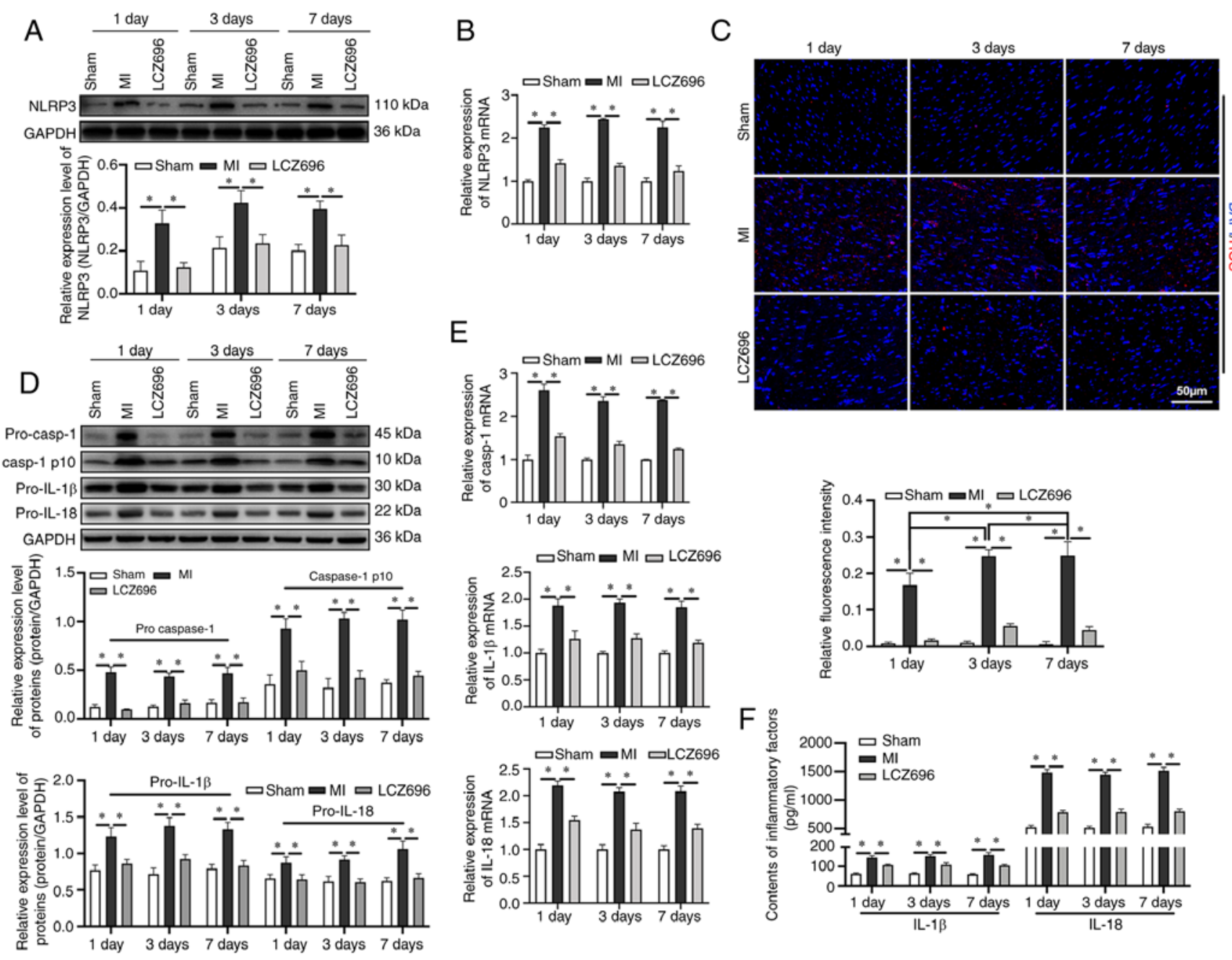

Figure 2. LCZ696 inhibits activation of the NLRP3 inflammasome, downstream inflammatory responses and oxidative damage. (A) Protein and (B) mRNA expression levels of the NLRP3 inflammasome, as detected by western blotting and reverse transcription-quantitative PCR. (C) Expression levels of reactive oxygen species, as detected by immunofluorescence (scale bar=50 $\mu \mathrm{m}$ ). (D) Relative expression levels of pro-caspase-1, caspase-1 p10, pro-IL-1 $\beta$ and pro-IL-18, as determined by western blotting. (E) mRNAs expression levels of caspase-1, IL-1 $\beta$ and IL-18. (F) Levels of inflammatory factors IL-1 $\beta$ and IL-18 in samples of rat serum determined using ELISA. Data are given as mean \pm standard deviation. "P $<0.05$. LCZ696, sacubitril/valsartan; NLRP3, NLR pyrin family domain containing 3 ; casp, caspase.
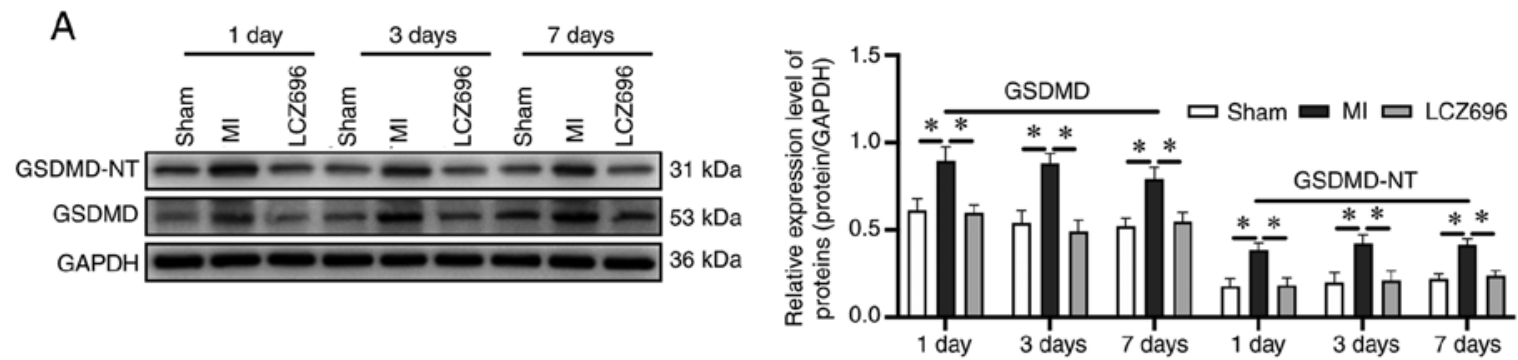

B

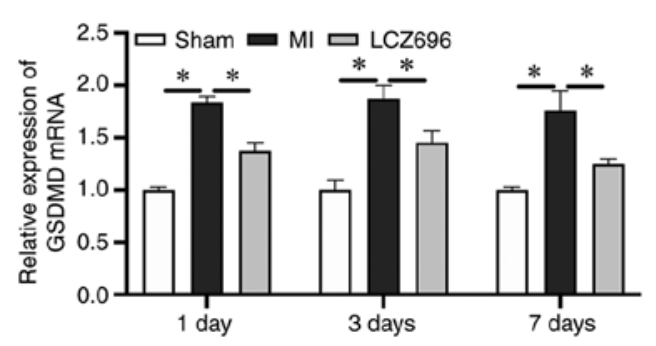

Figure 3. LCZ696 improves pyroptosis in a rat model of MI. (A) Protein expression levels of GSDMD-NT and GSDMD, as detected by western blotting. (B) mRNA expression levels of GSDMD, as determined by reverse transcription-quantitative PCR. Data are given as mean \pm standard deviation. ${ }^{*} \mathrm{P}<0.05$. LCZ696, sacubitril/valsartan; GSDMD, gasdermin D; -NT, N-terminal cleavage product; MI, myocardial infarction. 

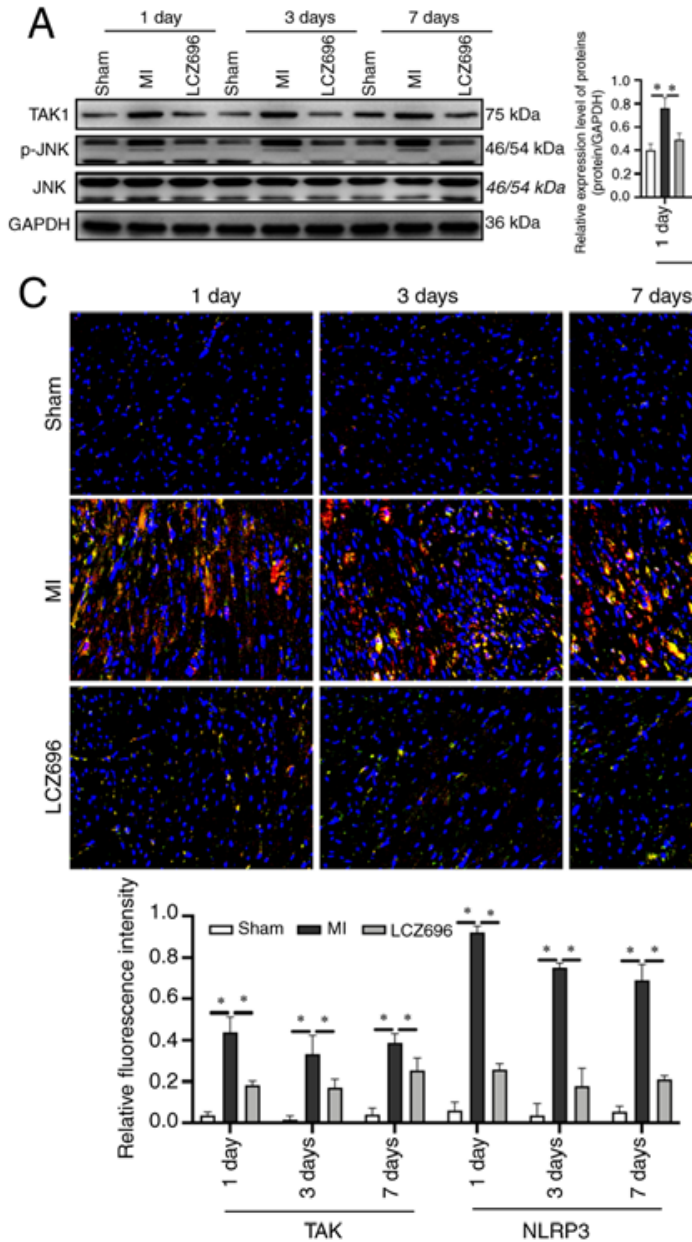

B
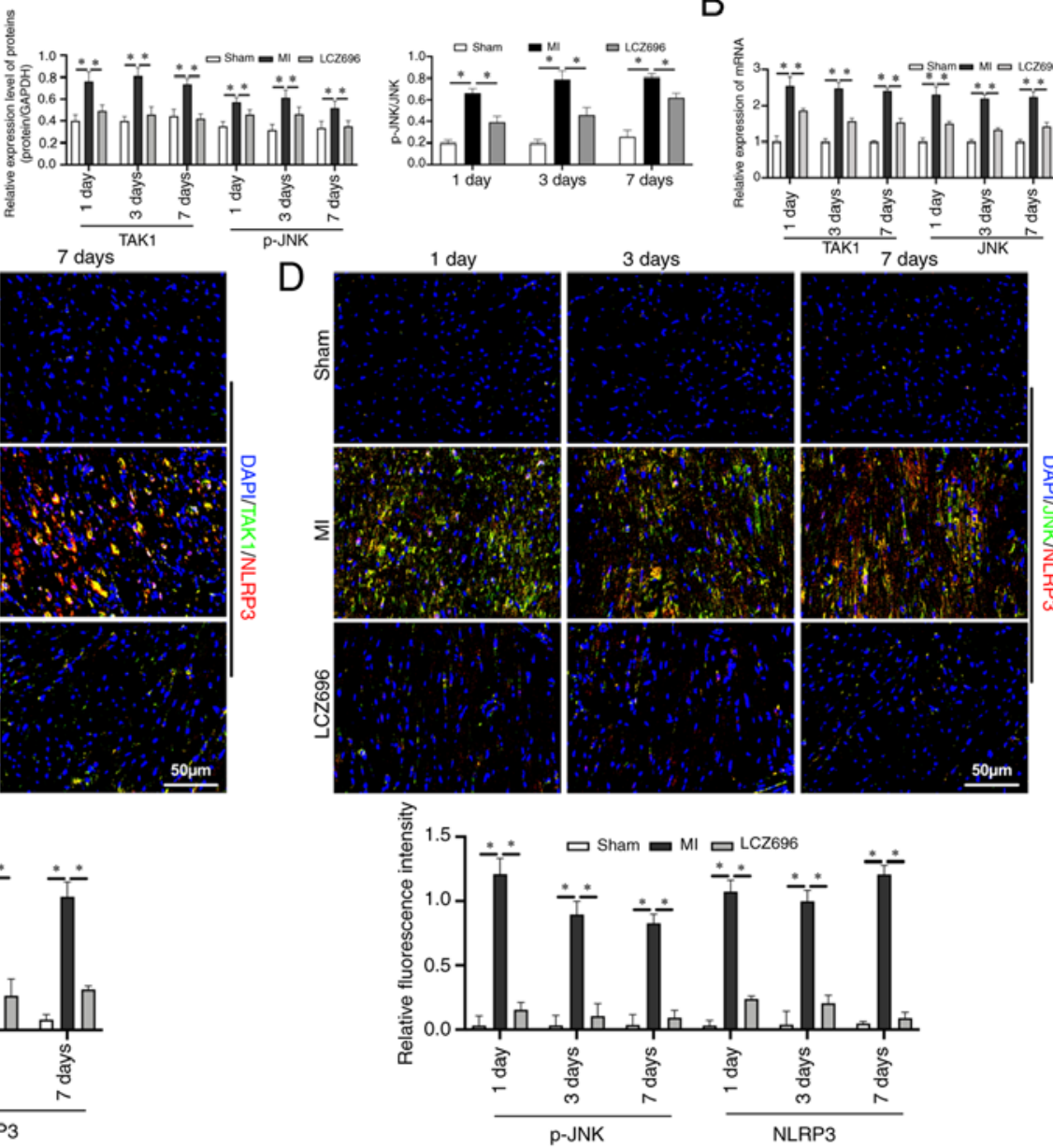

Figure 4. LCZ696 suppresses the activation of the NLRP3 inflammasome and inhibits the expression of proteins associated with the TAK1/JNK pathway in a rat model of MI. (A) Levels of TAK1 protein and phosphorylation of JNK (p-JNK) and p-JNK/JNK as detected by western blotting. (B) mRNA expression levels of TAK1 and JNK, as determined by reverse transcription-quantitative PCR. (C) Dual-fluorescent labelling staining of TAK1/NLRP3 (scale bar $=50 \mu \mathrm{m}$ ). (D) Dual-fluorescent labelling staining of p-JNK/NLRP3 (scale bar=50 $\mu \mathrm{m}$ ). Data are given as mean \pm standard deviation, ${ }^{*} \mathrm{P}<0.05$. LCZ696, sacubitril/valsartan; NLRP3, NLR pyrin family domain containing 3; TAK1, transforming growth factor $\beta$-activated kinase-1; p-, phosphorylated.

pathway. The expression levels of proteins in the TAK1/JNK pathway and NLRP3 in myocardial tissues was also investigated; it was found that both TAK1/NLRP3 and JNK/NLRP3 were co-expressed in myocardial tissues (Fig. 4C and D), suggesting that the TAK1/JNK pathway may mediate the expression of the NLRP3 inflammasome.

The TAK1/JNK pathway activates the NLRP3 inflammasome and pyroptosis. As the present study observed the co-expression of TAK1/NLRP3 in the rat model of MI, the role of TAK1 in NLRP3-induced myocardial cell pyroptosis was next investigated. A hypoxia-simulated model of MI was created in $\mathrm{H} 9 \mathrm{C} 2$ cardiomyocytes in vitro. It was found that the protein expression levels of TAK1 and the phosphorylation of JNK were both elevated in cell model of MI (Fig. 5A and B). However, LCZ696 was able to inhibit the effects on the NLRP3 inflammasome and pro-caspase- 1 in the in vitro studies. Notably, the overexpression of TAK1 reversed the inhibitory effects of LCZ696 on hypoxia-induced inflammatory responses in myocardial cells and this led to an increase in the in vitro expression and lysis of GSDMD, the activation of NLRP3 and an increase in the expression levels of pro-caspase-1 (Fig. 5A). It was also found that the levels of IL-1 $\beta$ and IL-18 were enhanced in cell supernatants (Fig. 5C), thus indicating that LCZ696 improved MI-induced inflammatory responses via the TAK1/JNK signaling pathway.

\section{Discussion}

Ventricular remodeling is a key physiological process following MI. Myocardial cells begin to die in the early stages of ventricular remodeling $(40,41)$. Myocardial systolic and diastolic function depends on the integrity of the cardiomyocytes (42). However, due to regenerative limitations, the death of a significant number of myocardial cells will inevitably lead to a decline in cardiac function. The present study established a model of MI model by ligating the left anterior descending coronary artery in SD rats and then treated these animals with LCZ696. It was found that LCZ696 improved MI-induced myocardial fibrosis, inhibited inflammatory responses and improved cardiac functions. Previous studies reported that the expression levels of NLRP3 increase during the early remodeling stage following MI and that NLRP3 can recruit and activate caspase- 1 by combining with ASC (43-45). The activation of caspase- 1 promotes the maturation and release of IL-1 $\beta$ which ultimately leads to the death of cardiomyocytes; 


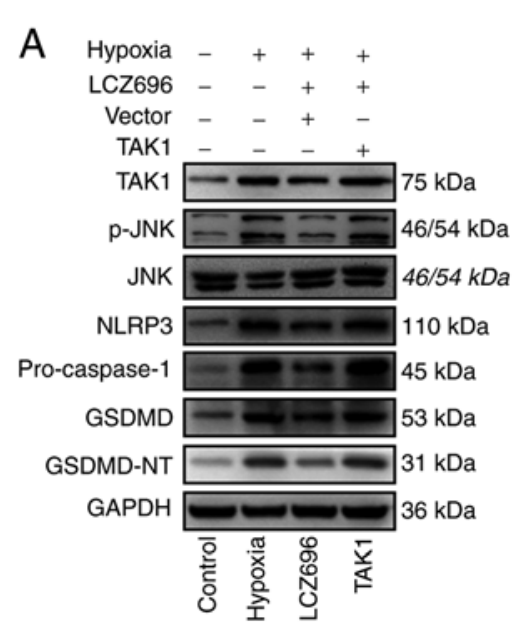

B
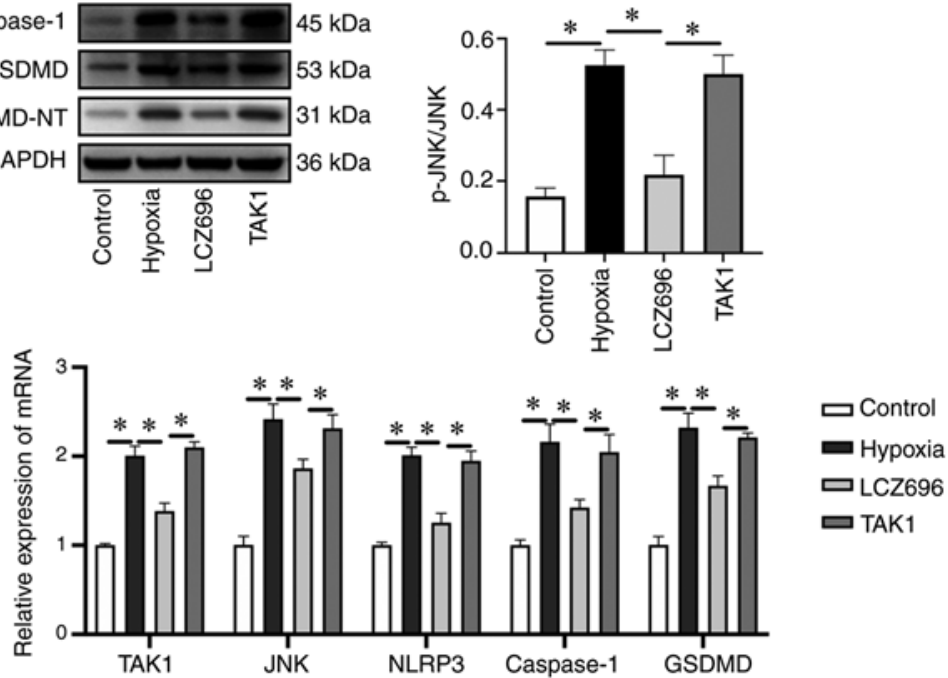

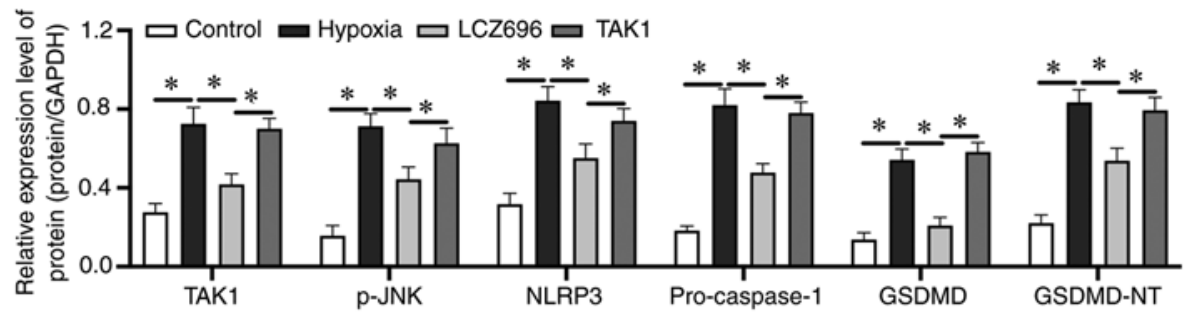

C

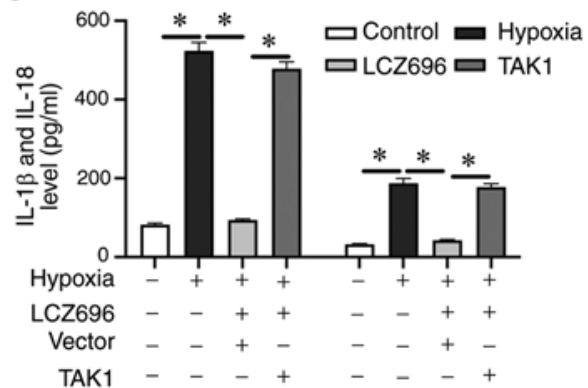

Figure 5. Activation of the TAK1/JNK pathway leads to accumulation of the NLRP3 inflammasome and the induction of cellular pyroptosis, resulting in increased inflammatory responses in $\mathrm{H} 9 \mathrm{c} 2$ cells. (A) Relative expression levels of proteins, as detected by western blotting. (B) mRNA expression levels of TAK1, JNK, NLRP3, caspase-1 and GSDMD, as determined by reverse transcription-quantitative PCR. (C) Levels of IL-1 $\beta$ and IL-18, in samples of rat serum, as detected by ELISA. Data were given as mean \pm standard deviation, ${ }^{\prime} \mathrm{P}<0.05$. TAK1, transforming growth factor $\beta$-activated kinase-1; NLRP3, NLR pyrin family domain containing 3; GSDMD, gasdermin D; p-, phosphorylated.

caspase-1 regulates Ang II-induced cardiomyocyte hypertrophy via the upregulation of IL-1 (46). TGF- $\beta$ is known to serve a crucial role in ventricular remodeling (47-50). The present study explored the association between the non-canonical TGF- $\beta$ signaling pathway TAK1/JNK and cell pyroptosis. It was found that over-activation of the NLRP3 inflammasome was associated with the death of cardiomyocytes by pyroptosis and that the TAK $1 / \mathrm{JNK}$ pathway can promote the activation of NLRP3. The inhibition of TAK1 and JNK led to a significant suppression of myocardial damage. Therefore, the targeted inhibition of TAK1 can block the process of pyroptosis in cardiomyocytes, thereby improving myocardial injury. Thus, TAK1 represents a potential novel therapeutic target.

LCZ696 is a novel drug that was designed for the treatment of cardiovascular diseases. It combines valsartan (an angiotensin receptor blocker) and sacubitril (an enkephalinase inhibitor prodrug) at a ratio of 1:1 in a sodium supramolecular complex (51). LCZ696 can considerably increase the levels of natriuretic peptide, which can exhibit diuretic and anti-proliferative effects and can exhibit effects against cardiac hypertrophy (51). In addition, LCZ696 can also reduce Ang II type 1 receptor activity, inhibit vasoconstriction and sympathetic nerve excitement, reduce the secretion of aldosterone and suppress inflammatory responses and oxidative stress by inhibiting enkephalinase and Ang II receptors $(52,53)$. A previous study compared the efficacies of LCZ696 and enalapril in the treatment of MI and illustrated that LCZ696 can significantly increase the rate of survival following MI and improve the imbalance of the RAAS and natriuretic peptide system, thus helping to prevent the myocardial complication of heart rupture after infarction (32). The in vivo experiments of the present study found that LCZ696 exhibited significant inhibition of myocardial fibrosis and improved ventricular remodeling. These data suggested that LCZ696 can inhibit pyroptosis and expression of the NLRP3 inflammasome in myocardial cells.

The death of cardiomyocytes is an essential mechanism during ventricular remodeling (54). Cellular apoptosis is an important mechanism underlying the death of cardiomyocytes (55). However, addressing apoptosis in cardiomyocytes has failed to identify a specific means of preventing cardiomyocyte death. As a newly discovered form of programmed cell death, cellular pyroptosis has received widespread attention because of its potential role in the occurrence and development of a variety of diseases (56-59). The present study investigated the expression levels of GSDMD and GSDMD-NT and found that pyroptosis serves an important role in the death of cardiomyocytes. Studies have also shown that inflammasomes such as NLRP3 and NLRP1 can chemotactically activate caspase-1 following extracellular stimulation, thus triggering the classic caspase-1-mediated cell pyroptosis pathway (60-62). Subsequently, the activated caspase-1 can cleave GSDMD at specific sites to release the GSDMD-N-terminal domain which can accumulate inside the cell membrane and form non-selective pores, thus resulting 
in an increase in the permeability of the cell membrane and leading to cellular pyroptosis (23). The present study demonstrated the dominant role of cell pyroptosis following MI and demonstrated that LCZ696 can suppress the expression levels of proteins related to the pyroptosis pathway. These data suggest that LCZ696 may improve ventricular remodeling by inhibiting NLRP3 pathways and cellular pyroptosis.

The NLRP3 inflammasome is an overall sensor of infection and stress; the activation of this inflammasome has been associated with a range of human diseases $(63,64)$. Research has demonstrated that TAK1 is the central regulator of NLRP3 inflammasome activation and spontaneous cell death (65). The lack of TAK1 in macrophages has been shown to induce the spontaneous activation of the NLRP3 inflammasome without TLR priming and subsequent activation signals, thus suggesting the unique role of TAK 1 in maintaining the steady state of the NLRP3 inflammasome (65-67). In a previous study, Malireddi et al (65). demonstrated that the loss of macrophage-specific TAK1 can lead to the uncontrolled secretion of TNF and abnormal signaling transmission, thereby leading to the spontaneous activation of the NLRP3 inflammasome. The present study first demonstrated that LCZ696 can inhibit the TAK $1 /$ JNK signaling pathway, thus indicating a potential mechanism by which this new drug exerts its effects. Furthermore, it confirmed that TAK $1 / \mathrm{JNK}$ is involved in the activation of the NLRP3 inflammasome. The overexpression of TAK1 was shown to regulate the level of JNK phosphorylation and simultaneously activated the NLRP3 inflammasome, thus promoting pyroptosis and the secretion of inflammatory factors. Notably, it was found that LCZ696 inhibited the expression of TAK1 and NLRP3. However, there are still some limitations to the present study. It would be more useful if whether LCZ696 could perform its effect on Myocardial injury after myocardial infarction under the activation of TAK1/JNK signaling pathway could be investigated.

In conclusion, the present study proposed that LCZ696 downregulated the expression of the NLRP3 inflammasome, reduced cell pyroptosis, improved ventricular remodeling and thus reduced myocardial damage by inhibiting the TAK1/JNK signaling pathway.

\section{Acknowledgements}

Not applicable.

\section{Funding}

The present study was supported by the National Key Research and development program (grant. no. 2016YFC1301004).

\section{Availability of data and materials}

The datasets used and analyzed during the current study are available from the corresponding author upon reasonable request.

\section{Authors' contributions}

JS and GQ designed the research, analyzed the data and wrote the manuscript. JS, ZF performed the experiments and prepared the figures. GS prepared the figures and analyzed the data. JS and GS confirmed the authenticity of all the raw data. GQ performed critical revision of the manuscript and supervised the study. All authors read and approved the final manuscript.

\section{Ethics approval and consent to participate}

The experiments were approved by the Laboratory Animal Welfare and Ethics Committee of China Medical University (approval number IACUC no. 2019109).

\section{Patient consent for publication}

Not applicable.

\section{Competing interests}

The authors declare that they have no competing interests.

\section{References}

1. Konstantinidis K, Whelan RS and Kitsis RN: Mechanisms of cell death in heart disease. Arterioscler Thromb Vasc Biol 32: 1552-1562, 2012.

2. Elmore S: Apoptosis: A review of programmed cell death. Toxicol Pathol 35: 495-516, 2007.

3. Cahill TJ and Kharbanda RK: Heart failure after myocardial infarction in the era of primary percutaneous coronary intervention: Mechanisms, incidence and identification of patients at risk. World J Cardiol 9: 407-415, 2017.

4. Bergsbaken T, Fink SL and Cookson BT: Pyroptosis: Host cell death and inflammation. Nat Rev Microbiol 7: 99-109, 2009.

5. Wree A, Eguchi A, McGeough MD, Pena CA, Johnson CD, Canbay A, Hoffman HM and Feldstein AE: NLRP3 inflammasome activation results in hepatocyte pyroptosis, liver inflammation, and fibrosis in mice. Hepatology 59: 898-910, 2014.

6. Man SM, Karki R and Kanneganti TD: Molecular mechanisms and functions of pyroptosis, inflammatory caspases and inflammasomes in infectious diseases. Immunol Rev 277: 61-75, 2017.

7. Azevedo PS, Polegato BF, Minicucci MF, Paiva SAR and Zornoff LAM: Cardiac remodeling: Concepts, clinical impact, pathophysiological mechanisms and pharmacologic treatment. Arq Bras Cardiol 106: 62-69, 2016.

8. Chen G, Li J, Song M, Wu Z, Zhang W, Wang Z, Gao J, Yang Z and $\mathrm{Ou} \mathrm{C}$ : A mixed component supramolecular hydrogel to improve mice cardiac function and alleviate ventricular remodeling after acute myocardial infarction. Adv Funct Materials 27: 1701798, 2017. https://doi.org/10.1002/adfm.201701798.

9. Kale P and Afzal A: Stage B heart failure: To strain or not to strain. JACC Cardiovasc Imaging 11: 1401-1404, 2018.

10. Bujak M and Frangogiannis NG: The role of TGF-beta signaling in myocardial infarction and cardiac remodeling. Cardiovasc Res 74: 184-195, 2007.

11. Dobaczewski MW, Chen W and Frangogiannis NG: Transforming growth factor (TGF)- $\beta$ signaling in cardiac remodeling. J Mol Cell Cardiol 51: 600-606, 2011.

12. Frangogiannis NG: The role of transforming growth factor (TGF)- $\beta$ in the infarcted myocardium. J Thorac Dis 9 (Suppl 1): S52-S63, 2017.

13. Monzen K, Hiroi Y, Kudoh S, Akazawa H, Oka T, Takimoto E, Hayashi D, Hosoda T, Kawabata M, Miyazono K, et al: Smads, TAK1, and their common target ATF-2 play a critical role in cardiomyocyte differentiation. J Cell Biol 153: 687-698, 2001.

14. Mihaly SR, Ninomiya-Tsuji J and Morioka S: TAK1 control of cell death. Cell Death Differ 21: 1667-1676, 2014.

15. Okada M, Matsuzawa A, Yoshimura A and Ichijo H: The lysosome rupture-activated TAK1-JNK pathway regulates NLRP3 inflammasome activation. J Biol Chem 289: 32926-3236, 2014.

16. Toldo S, Mezzaroma E, Mauro AG, Salloum F, Van Tassell BW and Abbate A: The inflammasome in myocardial injury and cardiac remodeling. Antioxid Redox Signal 22: 1146-1161, 2015. 
17. Zhaolin Z, Guohua L, Shiyuan W and Zuo W: Role of pyroptosis in cardiovascular disease. Cell Prolif 52: e12563, 2019.

18. Mezzaroma E, Toldo S, Farkas D, Seropian IM, Van Tassell BW, Salloum FN, Kannan HR, Menna AC, Voelkel NF and Abbate A: The inflammasome promotes adverse cardiac remodeling following acute myocardial infarction in the mouse. Proc Natl Acad Sci USA 108: 19725-19730, 2011.

19. Liu D, Zeng X, Li X, Mehta JL and Wang X: Role of NLRP3 inflammasome in the pathogenesis of cardiovascular diseases. Basic Res Cardiol 113: 5, 2018.

20. Ferrini A, Stevens MM, Sattler S and Rosenthal N: Toward regeneration of the heart: Bioengineering strategies for immunomodulation. Front Cardiovasc Med 6: 26, 2019.

21. McKenzie BA, Mamik MK, Saito LB, Boghozian R, Monaco MC, Major EO, Lu JQ, Branton WG and Power C: Caspase-1 inhibition prevents glial inflammasome activation and pyroptosis in models of multiple sclerosis. Proc Natl Acad Sci USA 115: E6065-E6074, 2018.

22. Sharma B, McLeland CB, Potter TM, Stern ST and Adiseshaiah PP: Assessing NLRP3 inflammasome activation by nanoparticles. Methods Mol Biol 1682: 135-147, 2018.

23. Fann DY, Santro T, Manzanero S, Widiapradja A, Cheng YL, Lee SY, Chunduri P, Jo DG, Stranahan AM, Mattson MP and Arumugam TV: Intermittent fasting attenuates inflammasome activity in ischemic stroke. Exp Neurol 257: 114-119, 2014.

24. Zhu ZD, Ye JY, Niu H, Ma YM, Fu XM, Xia ZH and Zhang X: Effects of microRNA-292-5p on myocardial ischemiareperfusion injury through the peroxisome proliferator-activated receptor- $\alpha /-\gamma$ signaling pathway. Gene Ther 25: 234-248, 2018.

25. Volpe M, Carnovali M and Mastromarino V: The natriuretic peptides system in the pathophysiology of heart failure: From molecular basis to treatment. Clin Sci (Lond) 130: 57-77, 2016.

26. Zablocki D and Sadoshima J: Angiotensin II and oxidative stress in the failing heart. Antioxid Redox Signal 19: 1095-1109, 2013.

27. Jones ES, Vinh A, McCarthy CA, Gaspari TA and Widdop RE: AT2 receptors: Functional relevance in cardiovascular disease. Pharmacol Ther 120: 292-316, 2008

28. Michel MC, Brunner HR, Foster C and Huo Y: Angiotensin II type 1 receptor antagonists in animal models of vascular, cardiac, metabolic and renal disease. Pharmacol Ther 164: 1-81, 2016.

29. Kompa AR, Lu J, Weller TJ, Kelly DJ, Krum H, von Lueder TG and Wang $\mathrm{BH}$ : Angiotensin receptor neprilysin inhibition provides superior cardioprotection compared to angiotensin converting enzyme inhibition after experimental myocardial infarction. Int J Cardiol 258: 192-198, 2018

30. Shirley $M$ and McCormack PL: Perindopril/amlodipine (Prestalia $\AA$ ): A review in hypertension. Am J Cardiovasc Drugs 15: 363-370, 2015

31. Campbell DJ: A review of perindopril in the reduction of cardiovascular events. Vasc Health Risk Manag 2: 117-124, 2006.

32. Ishii M, Kaikita K, Sato K, Sueta D, Fujisue K, Arima Y, Oimatsu Y, Mitsuse T, Onoue Y, Araki S, et al: Cardioprotective effects of LCZ696 (Sacubitril/Valsartan) after experimental acute myocardial infarction. JACC Basic Transl Sci 2: 655-668, 2017.

33. Chang PC, Lin SF, Chu Y, Wo HT, Lee HL, Huang YC, Wen MS and Chou CC: LCZ696 therapy reduces ventricular tachyarrhythmia inducibility in a myocardial infarction-induced heart failure rat model. Cardiovasc Ther 2019: 6032631, 2019.

34. Livak KJ and Schmittgen TD: Analysis of relative gene expression data using real-time quantitative PCR and the 2(-Delta Delta C(T)) method. Methods 25: 402-408, 2001

35. Li W, Li Y, Chu Y, Wu W, Yu Q, Zhu X and Wang Q: PLCE1 promotes myocardial ischemia-reperfusion injury in $\mathrm{H} / \mathrm{R} \mathrm{H} 9 \mathrm{c} 2$ cells and $I / R$ rats by promoting inflammation. Biosci Rep 39 BSR20181613, 2019

36. Shi J, Zhao Y, Wang K, Shi X, Wang Y, Huang H, Zhuang Y, Cai T, Wang $F$ and Shao F: Cleavage of GSDMD by inflammatory caspases determines pyroptotic cell death. Nature 526: 660-665, 2015.

37. Liu X, Zhang Z, Ruan J, Pan Y, Magupalli VG, Wu H and Lieberman J: Inflammasome-activated gasdermin D causes pyroptosis by forming membrane pores. Nature 535: 153-158, 2016.

38. Malireddi R, Kesavardhana S and Kanneganti TD: ZBP1 and TAK1: Master regulators of NLRP3 inflammasome/pyroptosis, apoptosis, and necroptosis (PAN-optosis). Front Cell Infect Microbiol 9: 406, 2019.
39. Orning P, Weng D, Starheim K, Ratner D, Best Z, Lee B, Brooks A, Xia S, Wu H, Kelliher MA, et al: Pathogen blockade of TAK1 triggers caspase-8-dependent cleavage of gasdermin D and cell death. Science 362: 1064-1069, 2018.

40. Bhatt AS, Ambrosy AP and Velazquez EJ: Adverse remodeling and reverse remodeling after myocardial infarction. Curr Cardiol Rep 19: 71, 2017

41. Li J, Cai SX, He Q, Zhang H, Friedberg D, Wang F and Redington AN: Intravenous miR-144 reduces left ventricular remodeling after myocardial infarction. Basic Res Cardiol 113: 36, 2018.

42. Caporizzo MA, Chen CY, Bedi K, Margulies KB and Prosser BL: Microtubules increase diastolic stiffness in failing human cardiomyocytes and myocardium. Circulation 141: 902-915, 2020.

43. Tang YS, Zhao YH, Zhong Y, Li XZ, Pu JX, Luo YC and Zhou QL: Neferine inhibits LPS-ATP-induced endothelial cell pyroptosis via regulation of ROS/NLRP3/caspase-1 signaling pathway. Inflamm Res 68: 727-738, 2019.

44. Gao R, Shi H, Chang S, Gao Y, Li X, Lv C, Yang H, Xiang H, Yang J, Xu L and Tang Y: The selective NLRP3-inflammasome inhibitor MCC950 reduces myocardial fibrosis and improves cardiac remodeling in a mouse model of myocardial infarction. Int Immunopharmacol 74: 105575, 2019.

45. Takahashi M: Role of NLRP3 inflammasome in cardiac inflammation and remodeling after myocardial infarction. Biol Pharm Bull 42: 518-523, 2019.

46. Bai Y, Sun X, Chu Q, Li A, Qin Y, Li Y, Yue E, Wang H, Li G, Zahra SM, et al: Caspase-1 regulates Ang II-induced cardiomyocyte hypertrophy via up-regulation of IL-1 $\beta$. Biosci Rep 38: BSR20171438,2018

47. Cohn JN, Ferrari R and Sharpe N: Cardiac remodeling-concepts and clinical implications: A consensus paper from an international forum on cardiac remodeling. Behalf of an international forum on cardiac remodeling. J Am Coll Cardiol 35: 569-582, 2000.

48. Schnee JM and Hsueh WA: Angiotensin II, adhesion, and cardiac fibrosis. Cardiovasc Res 46: 264-268, 2000.

49. Yamazaki T, Komuro I, Shiojima I and Yazaki Y: The renin-angiotensin system and cardiac hypertrophy. Heart 76 (Suppl 3): S33-S35, 1996

50. Brand T and Schneider MD: Transforming growth factor-beta signal transduction. Circ Res 78: 173-179, 1996.

51. da Silva PM and Aguiar C: Sacubitril/valsartan: An important piece in the therapeutic puzzle of heart failure. Rev Port Cardiol 36: 655-668, 2017 (In English, Portuguese).

52. Forte M, Madonna M, Schiavon S, Valenti V, Versaci F, Zoccai GB, Frati G and Sciarretta S: Cardiovascular pleiotropic effects of natriuretic peptides. Int J Mol Sci 20: 3874, 2019.

53. Lee NS and Daniels LB: Current understanding of the compensatory actions of cardiac natriuretic peptides in cardiac failure: A clinical perspective. Card Fail Rev 2: 14-19, 2016.

54. Ni J, Li Y, Xu Y and Guo R: Salidroside protects against cardiomyocyte apoptosis and ventricular remodeling by AKT/HO-1 signaling pathways in a diabetic cardiomyopathy mouse model. Phytomedicine 82: 153406, 2021

55. Xia P, Liu Y and Cheng Z: Signaling pathways in cardiac myocyte apoptosis. Biomed Res Int 2016: 9583268, 2016.

56. Jia C, Chen H, Zhang J, Zhou K, Zhuge Y, Niu C, Qiu J, Rong X, Shi Z, Xiao J, et al: Role of pyroptosis in cardiovascular diseases. Int Immunopharmacol 67: 311-318, 2019.

57. Wu J, Lin S, Wan B, Velani B and Zhu Y: Pyroptosis in liver disease: New insights into disease mechanisms. Aging Dis 10: 1094-1108, 2019

58. Guo H, Xie M, Zhou C and Zheng M: The relevance of pyroptosis in the pathogenesis of liver diseases. Life Sci 223: 69-73, 2019.

59. Zheng $\mathrm{Z}$ and Li G: Mechanisms and therapeutic regulation of pyroptosis in inflammatory diseases and cancer. Int J Mol Sci 21: $1456,2020$.

60. Teng JF, Mei QB, Zhou XG, Tang Y, Xiong R, Qiu WQ Pan R, Law BYK, Wong VKW, Yu CL, et al: Polyphyllin VI induces caspase-1-mediated pyroptosis via the induction of $\mathrm{ROS} / \mathrm{NF}-\kappa \mathrm{B} / \mathrm{NLRP} 3 / \mathrm{GSDMD}$ signal axis in non-small cell lung cancer. Cancers (Basel) 12: 193, 2020.

61. Li A, Yu Y, Ding X, Qin Y, Jiang Y, Wang X, Liu G, Chen X, Yue E, Sun X, et al: MiR-135b protects cardiomyocytes from infarction through restraining the NLRP3/caspase-1/IL-1 $\beta$ pathway. Int J Cardiol 307: 137-145, 2020.

62. Tan M, Tan L, Jiang T, Zhu XC, Wang HF, Jia CD and Yu JT: Amyloid- $\beta$ induces NLRP1-dependent neuronal pyroptosis in models of Alzheimer's disease. Cell Death Dis 5: e1382-e1382, 2014. 
63. Burdette D, Haskett A, Presser L, McRae S, Iqbal J and Waris G: Hepatitis $C$ virus activates interleukin-1beta via caspase-1-inflammasome complex. J Gen Virol 93: 235-246, 2012.

64. Theofani E, Semitekolou M, Morianos I, Samitas K and Xanthou G: Targeting NLRP3 inflammasome activation in severe asthma. J Clin Med 8: 1615, 2019.

65. Malireddi RS, Gurung P, Mavuluri J, Dasari TK, Klco JM, Chi $\mathrm{H}$ and Kanneganti TD: TAK1 restricts spontaneous NLRP3 activation and cell death to control myeloid proliferation. J Exp Med 215: 1023-1034, 2018.

66. Malireddi R, Gurung P, Kesavardhana S, Samir P, Burton A, Mummareddy H, Vogel P, Pelletier S, Burgula S and Kanneganti TD: Innate immune priming in the absence of TAK1 drives RIPK kinase activity-independent pyroptosis, apoptosis, necroptosis, and inflammatory disease. J Exp Med 217: 20191644, 2020.
67. Yang Y, Wang, Kouadir M, Song H and Shi F: Recent advances in the mechanisms of NLRP3 inflammasome activation and its inhibitors. Cell Death Dis 10: 128, 2019.

This work is licensed under a Creative Commons Attribution-NonCommercial-NoDerivatives 4.0 International (CC BY-NC-ND 4.0) License. 